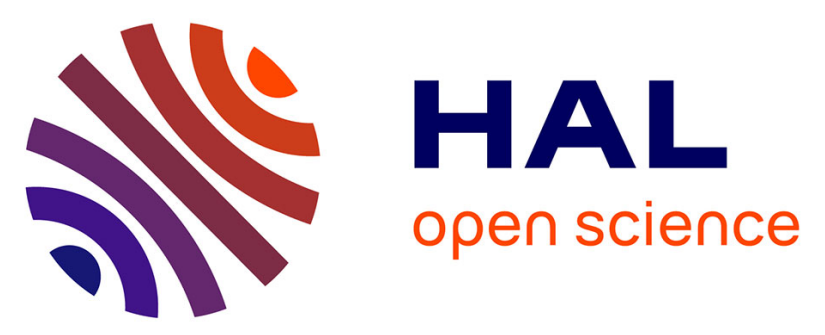

\title{
Hierarchical large-scale elastic metamaterials as an innovative passive isolation strategy for seismic wave mitigation
}

N. Kherraz, M. Miniaci, F. Bosia, A.S. S Gliozzi, M. Onorato, N.M. M Pugno

\section{- To cite this version:}

N. Kherraz, M. Miniaci, F. Bosia, A.S. S Gliozzi, M. Onorato, et al.. Hierarchical large-scale elastic metamaterials as an innovative passive isolation strategy for seismic wave mitigation. 14th International Congress on Artificial Materials for Novel Wave Phenomena, Metamaterials'2020, Sep 2020, New York City, NY, United States. pp.84-86, 10.1109/Metamaterials49557.2020.9285161 . hal-03209110

\author{
HAL Id: hal-03209110 \\ https://hal.science/hal-03209110
}

Submitted on 23 Dec 2021

HAL is a multi-disciplinary open access archive for the deposit and dissemination of scientific research documents, whether they are published or not. The documents may come from teaching and research institutions in France or abroad, or from public or private research centers.
L'archive ouverte pluridisciplinaire HAL, est destinée au dépôt et à la diffusion de documents scientifiques de niveau recherche, publiés ou non, émanant des établissements d'enseignement et de recherche français ou étrangers, des laboratoires publics ou privés. 


\title{
Hierarchical Large-Scale Elastic Metamaterials as an Innovative Passive Isolation Strategy for Seismic Wave Mitigation
}

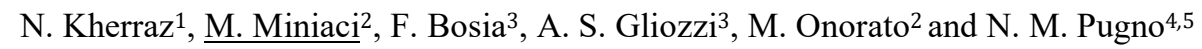 \\ 1 University of Torino, Department of Physics, Via Pietro Giuria 1, 10125, Torino, Italy \\ 2 CNRS, Centrale Lille, ISEN, Univ. Lille, Univ. Valenciennes, UMR 8520 - IEMN, F-59000 Lille, France \\ 3 Politecnico di Torino, Department of Applied Science and Technology, Corso Duca degli Abruzzi 24, \\ 10124 Torino, Italy \\ $4 \quad$ University of Trento, Laboratory of Bio-Inspired, Bionic, Nano, Meta Materials \& Mechanics, Department \\ of Civil, Environmental and Mechanical Engineering, Via Mesiano77, 38123 Trento, Italy \\ 5 Queen Mary University of London, School of Engineering \& Materials Science, Mile End Road, London \\ E1 4NS, UK \\ email of the presenter: marco.miniaci@gmail.com
}

\begin{abstract}
Large scale elastic metamaterials as an innovative passive isolation strategy for seismic waves has recently attracted increasing interest in the scientific community. In this work, we investigate the feasibility of an innovative design based on hierarchical organization of the unit cell, i.e. a structure having a self-similar geometry repeated at different scale levels. Results show how the introduction of hierarchy allows the conception of unit cells exhibiting reduced size while attaining good isolation efficiency at frequencies of interest for earthquake engineering.
\end{abstract}

\section{INTRODUCTION}

The design of buildings and structures capable of withstanding large scale vibrations has been the focus of researchers for many decades. This is particularly important for strategic facilities such as hospitals, skyscrapers, long span bridges, etc. Among all the possible sources of vibrations, earthquakes are the most devastating. Seismic waves are superposition of inhomogeneous acoustic waves with various wavelengths, comprising longitudinal and shear bulk waves, and surface acoustic waves, including Rayleigh and Love waves. Waves localizing at the interface are the most destructive because of their low frequencies, large amplitudes, and limited attenuation when propagating. Indeed, surface waves decay slower than bulk waves, resulting in the main cause for partial or even total collapse of buildings and structures [1].

Inthis context, althougha varietyof isolating systems (passive, active, hybridandsemi-active)have beendeveloped and shown to be effective in tests, some problems still exist with them and a commonly accepted method for the design of seismic-resistant buildings and structures has not yet been developed. To overcome these limitations, the idea of using phononic crystals for seismic mitigation purposes appeared for the first time in 1999 in the pioneering research of Meseguer et al. [2], who formulated for the first time the idea of drilling huge periodic hollows in the ground to attenuate surface seismic waves. In that occasion, the attenuation performance was experimentally tested in scaled experiments performed on a marble quarry in the $\mathrm{kHz}$ frequency range. After this pioneering intuition, many years passed without any further investigation in this direction, up to 2014, when Bru ^le' et al. [3] performed large scale experiments on meter-size periodic holes drilled in the ground, proving the practical attenuation potential of this approach in the $50 \mathrm{~Hz}$ frequency range. Afterwards, several approaches both based on large scale phononic crystals and resonant structures were proposed [4, 5], trying to meet requirements of lower (below $5 \mathrm{~Hz}$ ) frequency attenuation [6], practical manufacturing designs [4], and other technological constraints. In this context, reducing the size of the in-plane as well as the out-of-plane unit cells has been one of the challenges attracting great interest in the scientific community.

Here, we propose an innovative design strategy based on a hierarchical organization of the large scale unit cell. Results show that the introduction of hierarchy allows the conception of structures attaining isolation efficiency at lower frequencies if compared to the non hierarchical geometry, thus exhibiting reduced size. 


\section{MODEL DESCRIPTION}

Hierarchy is here understood in the sense that a representative unit cell comprises multiple arrangements of inhomogeneities at various size scales. If the same arrangement occurs at every scale, the pattern is called selfsimilar [7]. In this work, we consider drilling self-similar cross-like holes at two different hierarchical levels, as presented in Figs. 1A-C, in a sandy-type soil matrix with (Young's modulus $E_{\text {soil }}=20 \mathrm{MPa}$, Poisson's ratio $\nu_{\text {soil }}$ $=0.3$, mass density $\rho_{\text {soil }}=1800 \mathrm{~kg} \mathrm{~m}^{3}$ ) [4]. The corresponding geometrical parameters are summarised in Fig. 1E. Contrary to other studies, where the hierarchy was reproduced all over the non-hierarchical unit cell, here we introduce hierarchy only in specific regions (highlighted by the green arrows in Fig. 1B), since it was proved that these connecting regions were responsible for the main contribution to BG alteration [7].

A

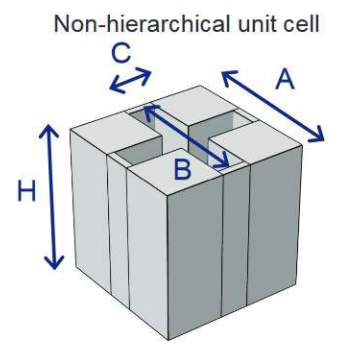

D

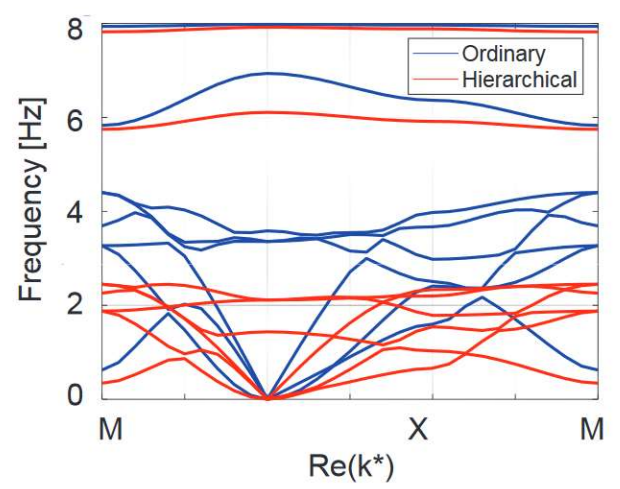

C
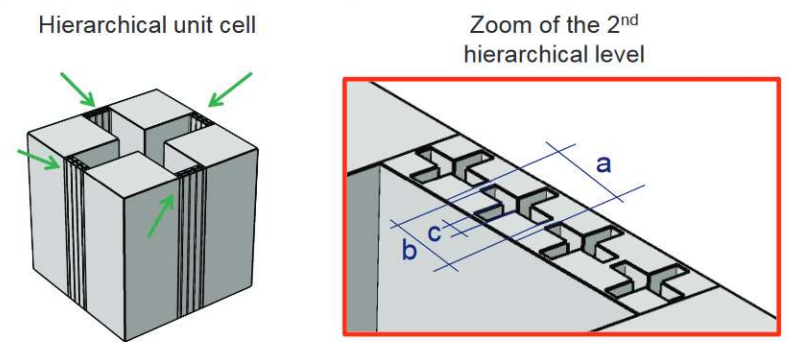

$\mathrm{E}$

\begin{tabular}{|l|l|}
\hline \multicolumn{2}{|c|}{ Geometrical parameters } \\
\hline $\mathrm{A}=5[\mathrm{~m}]$ & $\mathrm{a}=\mathrm{A} / \mathrm{HF}$ \\
\hline $\mathrm{B}=0.9 \mathrm{~A}$ & $\mathrm{~b}=0.9 \mathrm{a}$ \\
\hline $\mathrm{C}=0.2 \mathrm{~A}$ & $\mathrm{c}=0.2 \mathrm{a}$ \\
\hline \multicolumn{2}{|c|}{$\mathrm{H}=\mathrm{A}$} \\
\hline
\end{tabular}

Fig. 1: (A) Ordinary and (B) hierarchical large scale elastic metamaterial. (C) A zoom of the region where the second hierarchical level is introduced. (D) Comparison of dispersion curves of the ordinary (blue lines) and hierarchical (red lines) large scale elastic metamaterial. A remarkable shift of the BG towards lower frequencies is observable. (E) Geometrical parameters of the second hierarchical level. HF stands for hierarchical factor and is equal to 20 in this case.

\section{RESULTS}

The band structures are computed considering the unit cell to infinitely duplicate in the in-plane directions, assuming linear elastic behaviour for the soil, and adopting the standard Bloch-Floquet theory. The resulting eigenvalueproblemis solvedby varyingthe non-dimensionalwavevectork* alongthe irreduciblepath $[M-\Gamma-X-M]$, with $\Gamma \equiv(0,0), X \equiv \pi / a, 0)$ and $M \equiv \pi / a, \pi / a)$ being $a$ the lattice parameter [8].

Fig. 1D reports the band structures of the ordinary and hierarchical unit cells as blue and red lines, respectively. It 
is possible to observe that the hierarchy allows for a lower frequency band gap nucleation, although keeping the size of the unit cells unaltered. Specifically, a reduction of the operating BG frequency to half its original value is observed.

\section{CONCLUSION}

In conclusion, this study aims to contribute to a new generation of earthquake-proof barriers capable of inhibiting seismic waves before they reach the structures to be protected. We investigated the effect of hierarchy on the dispersion diagrams, showing its potential to conceive unit cells capable of nucleating frequency bandgaps below $5 \mathrm{~Hz}$ while keeping its unit cell size comparable to previous approaches. Results prove the strategy to be practical for civil structures.

\section{ACKNOWLEDGEMENT}

MM has received funding from the European Union's Horizon 2020 research and innovation programme under the Marie Sklodowska Curie grant agreement N. 754364. NK, MM, FB, ASG, MO have received funding from the project "Metapp" (No. CSTO160004) funded by Fondazione San Paolo and by the European Union's Horizon 2020 FET Open ("Boheme") under grant agreement No. 863179. NMP is supported by the European Commission under the Graphene Flagship Core 2 Grant no. 785219 (WP14 "Composites") and FET Proactive "Neurofibres" Grant no. 732344 as well as by the Italian Ministry of Education, University and Research (MIUR) under the "Departments of Excellence" Grant L.232/2016,the ARS01-01384-PROSCANGrant and the PRIN-20177TTP3S.

\section{REFERENCES}

[1] R. Villaverde, Fundamental Concepts of Earthquake Engineering, 2nd edition, Taylor \& Francis Group, 2009.

[2] F. Meseguer, M. Holgado, D. Caballero, N. Benaches, J. Sa'nchezDehesa, C. Lo'pez, and J. Llinares, "Rayleighwave attenuation by a semiinfinite twodimensional elasticbandgap crystal," Physical Review B, vol. 59, p. 12169, 1999.

[3] S. Bru^le', E. H. Javelaud, S. Enoch and S. Guenneau, "Experiments on seismic metamaterials: Molding surface waves," Physical Review Letters, vol. 112, p. 133901, 2014.

[4] M. Miniaci, A. Krushynska, F. Bosia and N. M. Pugno, "Large scale mechanical metamaterials as seismic shields," New Journal of Physics, vol. 18, p. 083041, 2016.

[5] A. Colombi, D. Colquitt, P. Roux, S. Guenneau and R. V. Craster, "A seismic metamaterial: The resonant metawedge," New Journal of Physics, vol. 18, p. 083041, 2016.

[6] Y. Achaoui, T. Antonakakis, S. Bru^le', R. V. Craster, S. Enoch and S. Guenneau, "Clamped seismic metamaterials: ultralow frequency stop bands," New Journal of Physics, vol. 19, p. 063022, 2017.

[7] M. Miniaci, A. Krushynska, A. S. Gliozzi, N. Kherraz, F. Bosia, and N. M. Pugno, "Design and Fabrication of Bioinspired Hierarchical Dissipative Elastic Metamaterials," Physical Review Applied, vol. 10, p. 024012, 2018.

[8] M. Miniaci, A. Marzani, N. Testoni and L. De Marchi, "Complete band gaps in a polyvinyl chloride (PVC) phononic plate with cross-like holes: numerical design and experimental verification,” Ultrasonics, vol. 56, p. 251-259, 2015. 